\title{
Identification of a novel gene HEPT3 that is overexpressed in human hepatocellular carcinoma and may function through its noncoding RNA
}

\author{
MEI CHUNG MOH${ }^{1}$, LAY HOON LEE ${ }^{1}$, XIAODONG YANG ${ }^{2}$ and SHALI SHEN ${ }^{1}$ \\ ${ }^{1}$ Laboratory of Hepato-Oncogenetics, Department of Physiology, Yong Loo Lin School of Medicine, \\ National University of Singapore, 2 Medical Drive, Singapore 117597; ${ }^{2}$ Department of Surgery, \\ No. 3 Hospital of Chongqing, Chongqing 400010, P.R. China
}

Received January 31, 2007; Accepted March 5, 2007

\begin{abstract}
Genetic alterations have been defined as the hallmark of cancers as they are responsible for the differences between normal and malignant phenotypes. A widely accepted approach to study genetic instability is to identify cancer-related genes, in particular, the two major groups of growth regulatory genes oncogenes and tumour suppressor genes. Using the technique of suppression subtractive hybridisation, we identified a novel gene transcript, designated as HEPT3. RT-PCR demonstrated that HEPT3 was overexpressed in $87 \%(20 / 23)$ of HCC patients and in 4/5 HCC cell lines tested. Sequence analyses performed on the full-length cDNA revealed that HEPT3 is an intronless gene mapped to human chromosome 6q13-14. The gene transcript lacks an extensive open reading frame and contains an Alu sequence near the 5' terminus, indicating that HEPT3 encodes a noncoding RNA. Antisense studies on the HCC cell line HepG2 showed that, when HEPT3 expression level was reduced, cell proliferation rate was inhibited by $\sim 5$-fold and cell colony formation was reduced by at least $50 \%$. Our data suggest that the novel gene HEPT3 may function through its noncoding RNA and its overexpression may play a role in hepatocarcinogenesis.
\end{abstract}

\section{Introduction}

Despite some advances in diagnosis and treatment, the prognosis of hepatocellular carcinoma (HCC) remains extremely poor accounting for almost one million deaths annually (1). The need of improving the overall outlook of HCC has urged scientists to delineate the yet mysterious mechanisms of hepatocarcinogenesis. Genetic alterations have

Correspondence to: Dr Shali Shen, Department of Physiology, Yong Loo Lin School of Medicine, National University of Singapore, 2 Medical Drive, Singapore 117597

E-mail:phsss1@nus.edu.sg

Key words: HEPT3, hepatocellular carcinoma, noncoding gene, intronless, antisense, cell growth been defined as the hallmark of cancers because they are responsible for the differences between normal and malignant phenotypes. A widely accepted approach to study genetic instability is to identify cancer-related genes, in particular, the two major groups of growth regulatory genes - oncogenes and tumour suppressor genes. The activation of oncogenes and inactivation of tumour suppressor genes play critical roles in the mechanisms of cancer development. These genes can induce malignant transformation when inappropriately expressed as a result of mutation, deletion, amplification or rearrangement. Studies on the genetic alterations in HCC have recently increased, reporting genes either up- or downregulated or lost in hepatocellular carcinoma (2-8). A considerable number of novel genes that are involved in hepatocarcinogenesis have hence emerged.

Exploring genes associated with HCC, we identified a novel gene, HEPT3, which is frequently overexpressed in HCC. HEPT3 is intronless and may function through its noncoding RNA. In this study, we report the identification and characterisation of the novel gene HEPT3 as well as the potential role of HEPT3 in liver tumorigenesis.

\section{Materials and methods}

Specimens and cell culture. A total of 23 pairs (tumour and non-tumour) of liver specimens and 2 pairs of colon specimens were collected after surgical resection from the No. 3 Hospital of Chongqing in China. Each sample was snap-frozen and stored in liquid nitrogen before experiment. The final diagnosis of HCC and colon cancer was confirmed by histological examination. The cell lines used in the study were mainly purchased from the American Type Culture Collection and maintained in the recommended media and culture conditions.

Suppression subtractive hybridisation. The technique of suppression subtractive hybridisation ( $\mathrm{SSH}$ ) was used to compare the mRNA profiles of HCC and its adjacent nontumourous liver tissues of a randomly chosen HCC patient. SSH was performed by using the PCR Select cDNA subtraction kit (Clontech, Palo Alto, CA, USA). The manufacturer's instructions were strictly followed. The SSH products, cDNA fragments representing the gene transcripts upregulated in 
Table I. Sequence information and applications of the primers.

\begin{tabular}{|c|c|c|c|}
\hline Primer & Sequence (from 5' to $3^{\prime}$ ) & PCR product (bp) & Application \\
\hline \multicolumn{4}{|l|}{$\mathrm{T} 3$} \\
\hline Forward & CAGTGAGAGTAGGCTTGTTTTAC & 281 & RT-PCR and real-time RT-PCR; \\
\hline Reverse & GGCCTCAAGACAACATAAGATAG & & ß-actin or GAPDH as internal control \\
\hline \multicolumn{4}{|l|}{ GAPDH } \\
\hline Forward & CGGATTTGGTCGTATTGGGC & 340 & \\
\hline Reverse & GGCAGAGATGATGACCCTTTG & & \\
\hline \multicolumn{4}{|l|}{ ß-actin } \\
\hline Forward & СТСТTCCAGCCTTCСТTCCT & 698 & \\
\hline Reverse & TGTGGACTTGGGAGAGGACT & & \\
\hline
\end{tabular}

T3

$\begin{array}{llll}\text { 5'RACE } & \text { GTCCGACAGAAGCCTCTACCGAGAACGT } & 1641 & \text { 5' RACE } \\ \text { nest5'RACE } & \text { CCTCATAGGGAGAAATCTTGCCAAGGGAG } & \end{array}$

T3

3'RACE CAGTGAGAGTAGGCTTGTTTTAC

$710 \quad$ 3' RACE

nest3'RACE CTATTTCTAGCCAGTGAGTTGTG

\begin{tabular}{|c|c|c|c|}
\hline \multicolumn{4}{|l|}{ T3full } \\
\hline Forward & ATTAGGAAACACTAGAATGGAA & 2613 & Cloning of the full-length \\
\hline Reverse & TCCTTATTTCAACAGTGTTCTTCTC & & cDNA into the pGEM-T vector \\
\hline \multicolumn{4}{|l|}{ AS } \\
\hline Forward & GctcgagaAAGGAACATAAAGGGTTAG & 1455 & Cloning of the HEPT3 antisense \\
\hline Reverse & Cgaattc ${ }^{a}$ TACTCCCAGTGTAACATAAG & & sequence into the pcDNA3.1 vector \\
\hline
\end{tabular}

${ }^{a}$ The letters in lower case are the sequences of the restriction enzyme cutting sites XhoI and EcoRI incorporated into the forward and the reverse primers, respectively.

HCC, were cloned into pGEM-T vector (Promega, Madison, WI, USA) and sequenced.

Rapid amplification of cDNA ends. Rapid amplification of cDNA ends (RACE) was employed to isolate the full-length cDNA of HEPT3. RACE was performed with the Human Liver Marathon-Ready cDNA kit (Clontech) using the HEPT3specific primers (Table I) in combination with the provided adapter primer AP1 or nested adapter primer AP2 to amplify the 5 ' and 3 ' portions of the HEPT3 transcript. The RACE products were cloned into pGEM-T vector and sequenced. Primers T3full-forward and T3full-reverse (Table I) at the 5'- and 3'-end of HEPT3 cDNA were then designed to generate the full-length cDNA by end-to-end PCR. The full-length cDNA was cloned into pGEM-T and sequenced.

Reverse transcription-polymerase chain reaction (RT-PCR). Semi-quantitative RT-PCR was performed with the OneStep RT-PCR kit (Qiagen, Hilden, Germany) while real-time RTPCR was performed with the LightCycler RNA amplification kit SYBR Green I (Roche, Basel, Switzerland). To determine

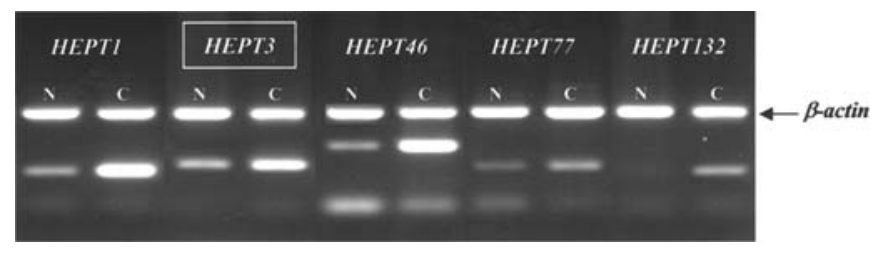

Figure 1. Evaluation of novel genes upregulated in an HCC patient by semiquantitative RT-PCR. HCC patient No. 8 was randomly chosen for SSH studies. The 5 novel genes identified by SSH were subjected to RT-PCR to confirm their differential expression in the same HCC patient. The RT-PCR products were analysed by gel electrophoresis. $\mathrm{N}$, non-tumourous liver tissue adjacent to the HCC; C, HCC tissue; $\beta$-actin, internal control. The designated name of each novel gene is indicated in the upper panel on the gel image. HEPT3 is highlighted in the box.

the mRNA level of HEPT3, primers T3-forward and T3-reverse (Table I) were used to generate a HEPT3 fragment from $0.2 \mu \mathrm{g}$ of DNase-treated total RNA. The OneStep RT-PCR conditions were: (1) $50^{\circ} \mathrm{C}$ for $30 \mathrm{~min}$; (2) $95^{\circ} \mathrm{C}$ for $15 \mathrm{~min}$; and (3) 35 cycles of $30 \mathrm{sec}$ at $94^{\circ} \mathrm{C}, 30 \mathrm{sec}$ at $55^{\circ} \mathrm{C}$, and $1 \mathrm{~min}$ at $72^{\circ} \mathrm{C}$; 


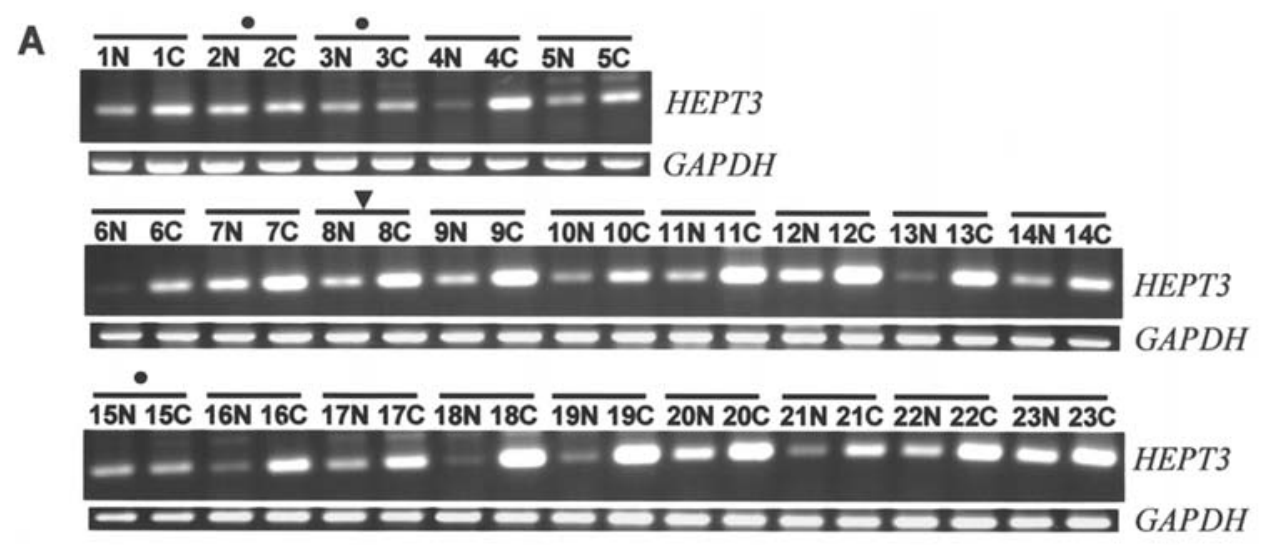

\section{B}

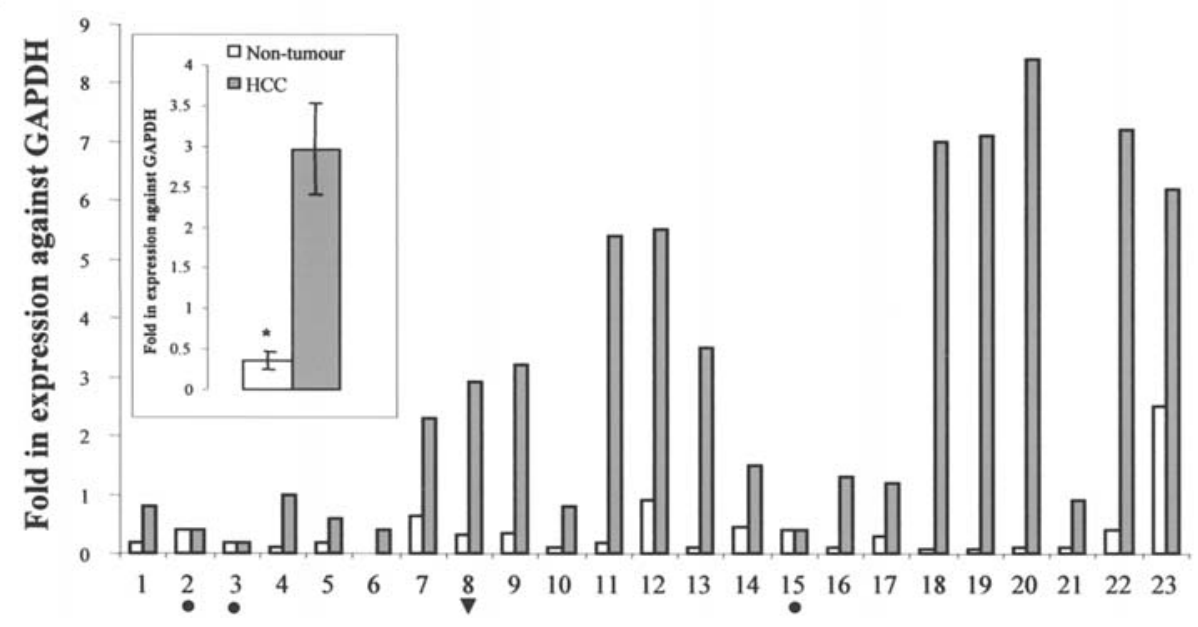

Figure 2. Overexpression of HEPT3 in HCC. (A) Screen of HEPT3 expression in 23 HCC patients. Semi-quantitative RT-PCR was performed to examine the differential expression of HEPT3 in paired liver specimens. The RT-PCR products were analysed by gel electrophoresis. N, non-tumourous liver tissue adjacent to the HCC; C, HCC tissue; samples marked by ' $\bullet$ ' did not show clear difference; ' $\boldsymbol{}$ ', sample chosen for SSH studies; GAPDH, internal control. (B) Quantitation of HEPT3 expression in 23 HCC patients. The expression level of HEPT3 in each sample was determined by real-time RT-PCR. Each result was normalised and presented as a fold increase in expression computed against the expression level of GAPDH in the same sample. The average expression level of HEPT3 is represented in the left inset (mean $\pm \mathrm{SD}, \mathrm{n}=23$ ). ${ }^{*} \mathrm{P}<0.0001$ as revealed by paired $\mathrm{t}$-test.

ended with $10 \mathrm{~min}$ at $72^{\circ} \mathrm{C}$. B-actin served as an internal control. RT-PCR products were analysed by gel electrophoresis. The real-time RT-PCR conditions were: (1) $55^{\circ} \mathrm{C}$ for $10 \mathrm{~min}$; (2) $95^{\circ} \mathrm{C}$ for $10 \mathrm{sec}$; and (3) for PCR amplification, 45 cycles of $5 \mathrm{sec}$ at $95^{\circ} \mathrm{C}, 10 \mathrm{sec}$ at $55^{\circ} \mathrm{C}$, and $12 \mathrm{sec}$ at $72^{\circ} \mathrm{C}$. The relative expression level of HEPT3 in each sample was presented in fold computed against the expression level of the internal control GAPDH.

Northern blot analysis. Total RNA $(40 \mu \mathrm{g})$ was separated on a $1 \%$ formaldehyde/MOPS [3-(N-morpholino) propanesulfonic acid] gel and transferred to Hybond- $\mathrm{N}^{+}$(Amersham, Germany) membrane by capillary blotting. Labeling of HEPT3 cDNA probe (nucleotides 645-1199) and hybridisation were carried out by using the DIG High Prime DNA labeling and detection starter kit I (Roche) according to the manufacturer's procedures.

Antisense plasmid construct and transfection studies. The antisense construct of HEPT3 was generated by PCR with primers AS-forward and AS-reverse (Table I), and cloned into the pcDNA3.1 vector (Invitrogen, Carlsbad, CA, USA). Transfections were carried out with the Lipofectamine Plus Reagents (Invitrogen). To establish stable transfection, HepG2 cells transfected with either the antisense HEPT3 plasmid or pcDNA3.1 vector were selected in the medium containing $800 \mu \mathrm{g} / \mathrm{ml}$ of G418 (Sigma, St. Louis, MO, USA) for 1 month, and then cloned and propagated. Real-time RT-PCR was performed to examine the mRNA levels of HEPT3 in a number of cell clones. Three cell clones, namely HEPT3-AS1, HEPT3-AS2 and HEPT3-AS3, with a significant reduction of HEPT3, were selected for further study. Two cell clones with pcDNA3.1 vector and the parental cells served as controls. To assess the rate of cell proliferation, $\sim 2 \times 10^{4}$ cells were seeded and cultured in 6-well plates. At every $24 \mathrm{~h}$ for 5 days, both microscopic analysis and MTT [3-(4,5-dimethylthiazol-2-yl)2,5-diphenyltetrazolium bromide] assay were performed to evaluate the cell density and viability, respectively. To establish the base line of cell proliferation, MTT assay was carried out once the cells had attached to the culture dishes on the day of plating. The growth rate of each cell line was presented as a fold increase in cell viability against the respective base line. 


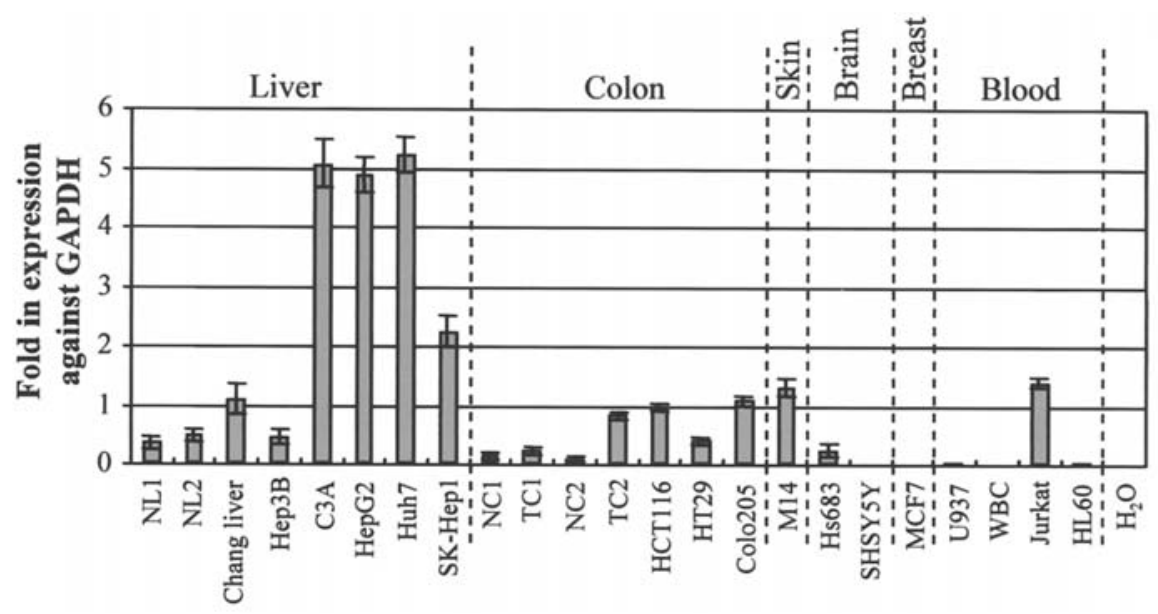

Figure 3. Expression level of HEPT3 in a variety of human tumours. Real-time RT-PCR was performed to determine the mRNA levels of HEPT3 in tissues and cell lines derived from different tumour types. The relative expression level of HEPT3 is presented in fold computed against the expression level of GAPDH in each sample (means $\pm \mathrm{SD}, \mathrm{n}=3$ repeats). The name and tissue type of each cell line are indicated at the bottom and the top of the graph, respectively. NL, normal liver tissue; $\mathrm{NC}$, normal colon tissue; TC, colon cancer tissue; WBC, normal white blood cells; $\mathrm{H}_{2} \mathrm{O}$, negative control.

Table II. Correlation between HEPT3 overexpression and the clinicopathological parameters in $23 \mathrm{HCC}$ patients. ${ }^{\mathrm{a}}$

\begin{tabular}{|c|c|c|c|c|}
\hline Parameters & $\begin{array}{c}\text { HEPT3 } \\
\text { overexpression }\end{array}$ & $\begin{array}{c}\text { HEPT3 } \\
\text { unchanged }\end{array}$ & $\begin{array}{c}\text { Overexpression } \\
\text { rate }(\%)\end{array}$ & $\mathrm{P}$ \\
\hline Total number & 20 & 3 & 87 & \\
\hline \multicolumn{5}{|l|}{ Sex } \\
\hline Male & 16 & 2 & 89 & NS \\
\hline Female & 4 & 1 & 80 & NS \\
\hline \multicolumn{5}{|l|}{ Grade } \\
\hline Well & 3 & 0 & 100 & NS \\
\hline Moderate & 11 & 3 & 79 & NS \\
\hline Poor & 6 & 0 & 100 & NS \\
\hline Cirrhosis & 16 & 3 & 84 & NS \\
\hline \multicolumn{5}{|l|}{ Hepatitis virus } \\
\hline HBV & 15 & 3 & 83 & NS \\
\hline $\mathrm{HCV}$ & 3 & 0 & 100 & NS \\
\hline $\mathrm{HBV}+\mathrm{HCV}$ & 2 & 0 & 100 & NS \\
\hline
\end{tabular}

aThe identities and personal data of all the patients are strictly kept confidential. Grade, histological differentiation of HCC. NS, not significant; $P$ was revealed by the Fisher's exact test.

For colony formation, HepG2 cells transfected with antisense HEPT3 construct or the vector alone were cultured in the medium containing $800 \mu \mathrm{g} / \mathrm{ml}$ of G418 for 3 weeks without trypsinisation. The cell colonies formed at the end of experiment were visible and the number of colonies in each plate was quantitated.

Bioinformatics and statistical analysis. Bioinformatics was carried out through database searches facilitated by the National Center for Biotechnology Information (NCBI), and motif searches with the RepeatMasker program (9). Software
InStat 3.0 (GraphPad, San Diego, CA, USA) was use for statistical analysis. For a small sample size $(n=6)$, the MannWhitney test was performed to compare two means while the nonparametric ANOVA for more than two means. Paired t-test was used to assess data with sample size $>6$. The Fisher's exact test was used to reveal the correlation between HEPT3 overexpression and the clinicopathological parameters in 23 HCC patients. $\mathrm{P}<0.05$ was considered significant.

\section{Results}

Identification of the novel gene HEPT3 in hepatocellular carcinoma. Using the technique of suppression subtractive hybridisation, we compared mRNA expression profiles of $\mathrm{HCC}$ and its adjacent non-tumourous liver tissue obtained from a randomly chosen HCC patient (No. 8 in Fig. 2A), and cloned 134 transcripts that were upregulated in HCC (data not shown). Five novel genes were identified as they did not have significant homology to known genes when subjected to database search. The differential expression of the five genes was confirmed by RT-PCR in the same HCC patient (Fig. 1). However, when screened against a panel of patients, only HEPT3 appeared to be constantly upregulated in HCC and was therefore chosen for further investigations.

Frequent upregulation of HEPT3 in HCC. Semi-quantitative RT-PCR was used to examine the expression of HEPT3 in 23 HCC specimens. The results showed that the expression of HEPT3 was elevated in $87 \%(20 / 23)$ of the HCC samples (Fig. 2A). Real-time RT-PCR was performed to determine the mRNA levels of HEPT3 in the liver specimens. In addition to the reproducible frequency of HEPT3 overexpression in HCC, real-time RT-PCR revealed an average of 2.5-fold upregulation of HEPT3 in HCCs compared to their adjacent non-tumourous liver tissues $(\mathrm{P}<0.0001$; Fig. 2B). Moreover, real-time RT-PCR analysis was performed in tissues and cell lines of a variety of human malignancies, including cancers of the liver, colon, skin, brain, breast and blood (Fig. 3). The results showed an overexpression of HEPT3 in 4 of the 5 
A

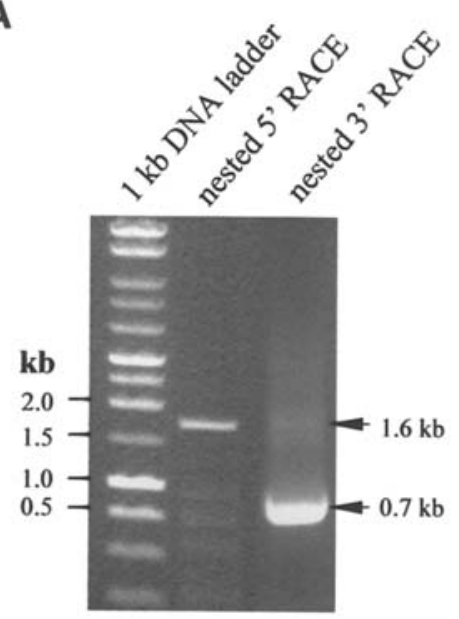

B

1 ATTAGGAAACACTAGAATGGAAAGGCCATTGGAAGACAGGTTGTATCTTTTTTAGACCATATTTCCTTGTTMAAAACTATCATTTGAATACTTTTTTGG 101 TGAAGAACTCCATGTTTTCAAGTTAAAGGTCACCTCGTAGGCCAGGCGCAGTGGCTCATGCCTGTAATCCCAGCACTCTGGGAGGCTGAGGCGGGTGAAT 201 CACAAGGTTAGGAGTTTGAGACCAGCCTGGCCAATATGGTGAAACCCCGTCCCTACTAAAAATACAAAATTTAGCCAGGCGTGGTGGCATGCACCTGTAG 301 rCCCACCTACTCGGGAGACTGAGGCAGGAGAATCACTTGAACCTGAGAGACAGAGGTTGCAGTGAGCCGAGP TCACGCCACTGCACTCCAGCCTGGGGGA 401 CAGAGTGAGATTCTGTCTCAAAAAACAAAAAACAAAAAAGTCACCTTGTAACTCATCTCTTTTTATTGTAAGTTTATTAAAAATGAAGAGGACAACAATG 501 AGAAGGAACATAAAGGGTTAGCTAGCACTGTCTCCTGGTGCATGGGGCTGTGCAGATGTCCCGGCCACTTCTTCCTTCATACTTCCCTTAGAGAACTTGC 601 TCTGCTACAAGCAGTGGGCTTGGACTAAAAGTGATTAAAATACCACAGGCATAAGGAGAAAAGGAGTATATGTAGTAGTAATAATTACTAGTATAAATTA 701 TTTTCTTCACATGCTATGAGTAATAATATTAAAAAACTCATTTTACCATTAAGATTCCTTATGCTGAAGCTCTTCCATTTAGAATACTGTCAATGTCATT 801 TACTGGTATGAACTAAAGTCCCCCTTCTTTTCCACTCACTGGGAACCTTAGTAAAACACCAGCATATCTTACCTCTCTTTCTGACTGGCCGATGCTTCCA 901 GAGACTGAATGTTGGGAAAACCTAGTAGCCAAACAATTCTAGGACAGAATAACATTTTTATATTTGGTTCCACCATCTTATTACATTTAGTTATAGTTTT 1001 AAAAAAGAAATTCAAGCCCATTAAAATATGTCTGGTCAATGAAATGCTTCCTTTTATTGTGTTGTGCTATTGTACTTTGTTTTTCAAAACATTGTAAAAA 1101 TAGTATCTTTGGTTTAGTATTTTGGATTATATATTATAATCTGAGGAGTGTCTGCTTATGTAGAATCCAGATATATTTCTGTTACCTAGGAGATGTTACT 1201 TACATATGTAATACTGTATCCTGCACGTGGAAATATTCAGAATTGTAGATAGCATAACTCTCCCTGCTCCTATTCTTTTGAGCCTAGGTATAATTTTTTT 1301 TTTTTTTTTAGAAAAAGACATATTTAGCTTTAATTTCTATTTATGCTAAACATATTTATAAGTAGTCTGTCAATATAATACCAACTATTTTTATTTTTAC 1401 ATAATTCAATTATTTCATTTGACATGTCTGGCAGACTCAAGACATTAAGTAAAAAATTGGAACTATGATTTTTCTTTGTCATTTTTTAAAAAAGAATTAT 1501 TTTATTAACCTGCTGGCATATAATCTGGAGTTCTTTTCACAACCTTACTTTTTCTGATTTGCTTTATTGAATGATTGAATACTCATTTCTTTCTAAAAAT 1601 ATGTTGTAAATTCTCCCTTGGCAAGATTTCTCCCTATGAGGGTAGTTATTATTTGAGTCTGCCAAGTGGTTACCATGGGGCAAGGTGCCATGATGTATTC 1701 TTGGGTGCATTGGTTTTTTGCGCATT' RACE 1801 GCTTCTGTCGGACAGGCAGAAGAGTGTATTCCTCACTTTTTTTTTTGTCTTCAAATTCCAGTAAGGCATAGCACTTTTAAGAAATTAGAATTTTTCTATC 1901 ATCTATGCAAATGATATTMATGTTAATATTAAATATCTTATGTTACACTGGGAGTAATTTGAGGTGCAATTATTTTTATTACTACTTTGAATAGAGGACC 2001 ATTATCCTTCTTTCTTCAGAAAACTAAGAAGTAAGTGTAACTTTTAAAGTAAGTATATATCAGTGAGAGTAGGCTTGTTTTACAACTATTTCTAGCCAGT 2101 GAGTTGTGTTTTCATGTCTCATCAAAAGACAATACCACATTGCATCATTTTACAAAATATGTTGTCATTTTCATTTCAGTTGTAACATAGGAAAATAGAT 2201 ATTTCCTAGATGATTTCTGAGTTTCTTACTGCAAAGAACAGTTATAAATTGGTATACATGTGTCTCTGTAATAGGGATAATATTGATATATCTGTTGCTA 2301 CATATTCAAGAATCATTCTATCTTATGTTGTCTTGAGGCCAAGATTTACCACGTTTGCCCAGTGTATTGAATTGGTGGTAGAAGGTAGTTCCATGTTCCA 2401 TTTGTAGATCTTTAAGATTTTATCTTTGATAACTTTAATAGAATGTGGCTCAGTTCTGGTCCTTCAAGCCTGTATGGTTTGGATTTTCAGTAGGGGACAG 2501 TTGATGTGGAGTCAATCTCTTTGGTACACAGGAAGCTTTATAAAATTTCATTCACGAATCTCTTATTTTGGGAAGCTGTTTTGCATATGAGAAGAACACT 2601 GTTGAAATAAGGAACTAAAGCTTTATATATTGATCAAGGTGATTCTGAAAGTTTTAATTTTTAATGTTGTAATGTTATGTTATTGTTAATTGTACTTTAT 2701 TATGTATTCAATAGAAAATCATGATTTATTAATAAAAGCTTAAATTCTCATCTAAAAAAAAAAAAAAAA $(2770)$

Figure 4. Isolation of full-length cDNA of HEPT3. (A) Nested 5' and 3' RACE products were analysed by gel electrophoresis. (B) Full-length cDNA sequence of HEPT3. The full-length cDNA of HEPT3 is $\sim 2.7 \mathrm{~kb}$. The Alu sequence is boxed, the mRNA destabilisation motif, ATTTA, is in bold, and the polyadenylation signal, AATAAA, is underlined. The primers used in RACE are indicated by arrows. The fragment of 700 bp generated by SSH is shaded.

HCC cell lines, but the upregulation of HEPT3 appeared significantly less in tissues and cell lines derived from other tumour types. The data indicate that HEPT3 overexpression is common in HCC, suggesting that the gene may be associated with the tumorigenesis of hepatocytes. However, no significant correlations were observed between HEPT3 overexpression and the clinicopathologic parameters in the 23 HCC patients (Table II). 

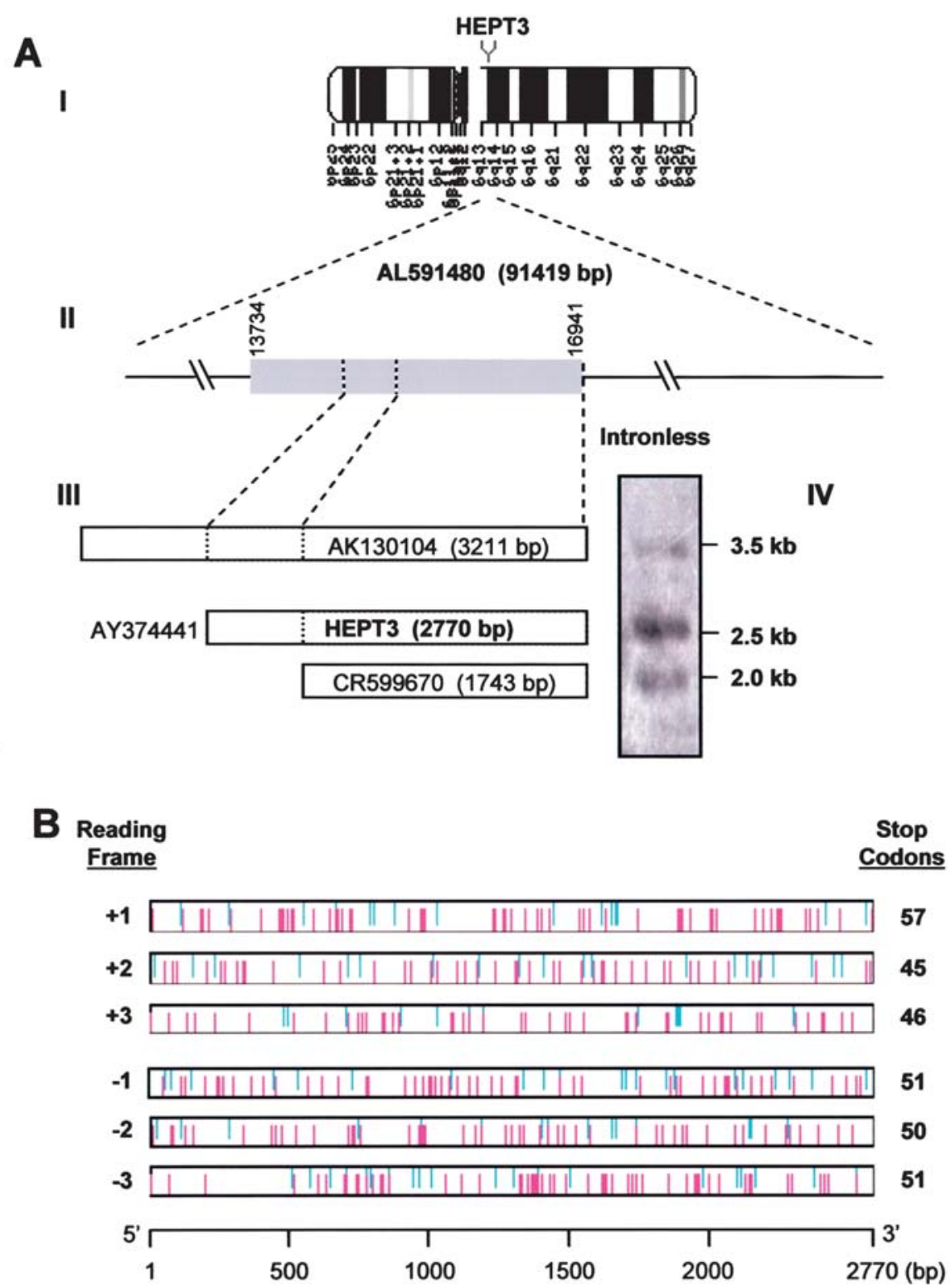

Figure 5. Characteristics of HEPT3. (A) Structure and isoforms of HEPT3. (I) HEPT3 is located on human chromosome 6q13-14. (II) The genomic DNA of HEPT3 (GenBank AL591480) lacks introns. (III) Two isoforms of HEPT3, AK130104 and CR599670, were retrieved from the database. (IV) Northern blot analysis of HEPT3 and the 2 isoforms. (B) Noncoding potential of HEPT3. Open reading frame was predicted and analysed in six possible frames. The high frequency of the stop codons on each frame is indicated by the small bars on the lower line and reflected in the right panel.

Sequence analysis of HEPT3 full-length cDNA. Four HEPT3 gene-specific primers designed on the fragment of $700 \mathrm{bp}$ obtained by SSH, as indicated in Fig. 4B, were used in the two consecutive rounds of 5' and 3' RACE reactions. The respective RACE products, $\sim 1.6 \mathrm{~kb}$ and $\sim 0.7 \mathrm{~kb}$ fragments (Fig. 4A), were cloned and sequenced. The overlapping sequences of the SSH and the 5' and 3' RACE products were aligned to obtain the full-length cDNA of HEPT3 of $\sim 2.7 \mathrm{~kb}$ (GenBank AY374441). As shown in Fig. 4B, the sequence contained a consensus polyadenylation signal, AATAAA, $23 \mathrm{bp}$ upstream of the poly(A) tail at the 3 ' terminus. Eleven copies of the mRNA destabilisation motif, AUUUA (ATTTA in the cDNA sequence) were identified throughout the sequence. Furthermore, through the RepeatMasker program (9), a 300-bp long Alu repetitive element was identified between the nucleotides 73 and 372 on the HEPT3 cDNA sequence.
HEPT3, an intronless gene mapped to chromosome 6q13-14. To determine the localisation of HEPT3 on human chromosomes, the HEPT3 full-length cDNA sequence was subjected to searches against the human genome database of the NCBI algorithm. The results showed that HEPT3 was specifically mapped to the human chromosome 6q13-14 (Fig. 5AI). Comparative analysis showed that HEPT3 cDNA was collinear to the genomic sequence (GenBank AL591480), indicating that the gene HEPT3 is intronless (Fig. 5AII).

Northern blot analysis was performed using a cDNA probe without the HEPT3 Alu element to avoid non-specific hybridisation. The results revealed three differently sized signals of $\sim 3.3,2.7$ and $2.0 \mathrm{~kb}$ (Fig. 5AIV), suggesting that HEPT3 may have isoforms. Indeed, database analysis revealed two uncharacterised full-length cDNAs that matched both the cDNA sequence and the chromosomal location of HEPT3 
A

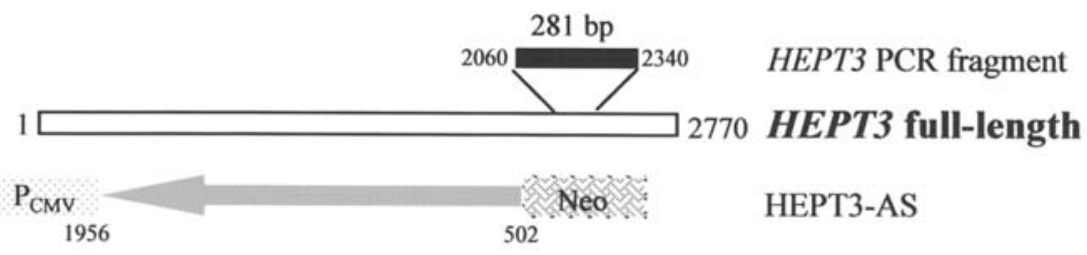

B
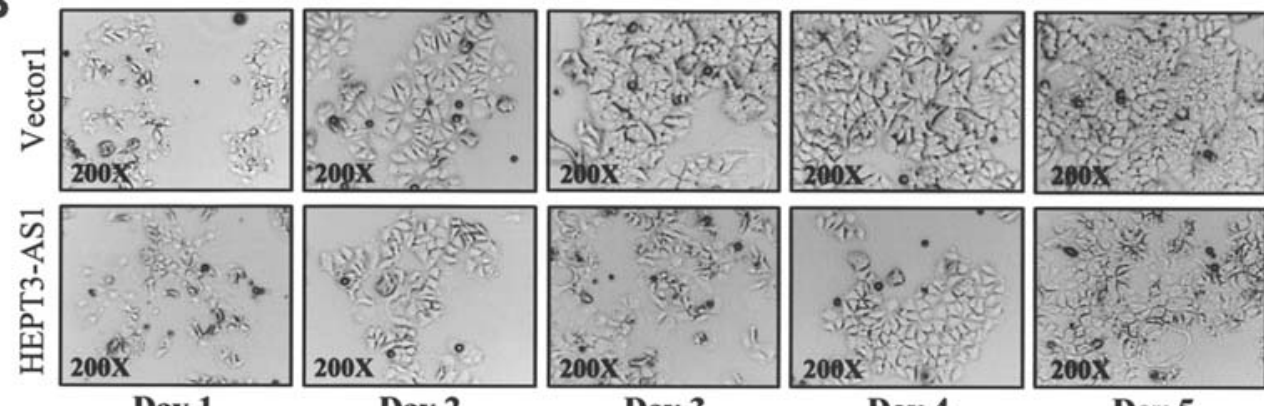

Day 2

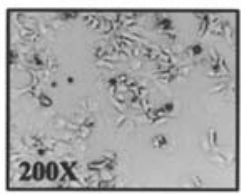

Day 3

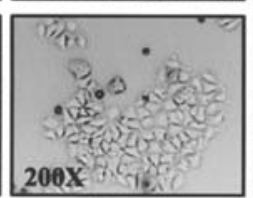

Day 4

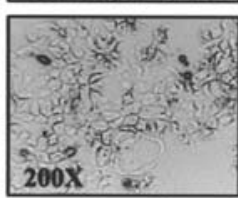

Day 5
C

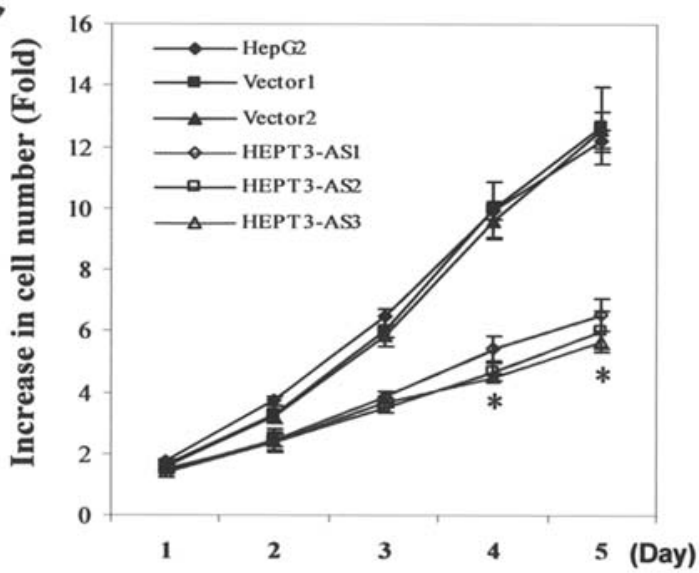

E

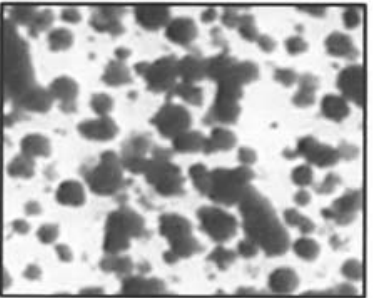

Vector
D

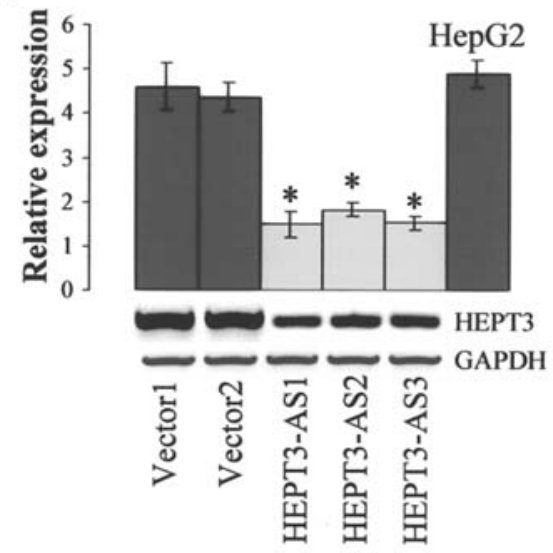

Figure 6. Antisense studies. (A) Schematic representation of HEPT3 antisense construct, namely HEPT3-AS. The construct was generated by cloning a fragment of HEPT3 cDNA (from 502 to 1956) in the reverse order into the pcDNA3.1 vector. The forward and reverse HEPT3 gene-specific primers located at 2060 and 2340, respectively, were used to generate the PCR product of $281 \mathrm{bp}$. (B and C) Analysis of cell growth rate. Three cell clones stably transfected with HEPT3 antisense construct were compared to two cell clones with pcDNA3.1 vector and the parental cells for their growth rate. At every 24 h for 5 days, both microscopy (B) and MTT assay (C) were performed to evaluate cell density and viability, respectively. The growth rate is represented as a fold increase in cell viability (means $\pm \mathrm{SD}, \mathrm{n}=6$ ); ${ }^{*} \mathrm{P}<0.001$ as revealed by nonparametric ANOVA. (D) Suppression of endogenous HEPT3 evaluated by real-time RT-PCR. The expression level of HEPT3 is presented in fold against the expression level of GAPDH in each sample (means $\pm \mathrm{SD}$, $\mathrm{n}=3$ repeats); ${ }^{*} \mathrm{P}<0.001$ as revealed by nonparametric ANOVA. The PCR product was examined by gel electrophoresis for its specificity (under each bar). (E) Colony formation. The size and density of cell colonies are shown in the photographs. The number of colonies on each plate is represented in the bar chart $(\mathrm{means} \pm \mathrm{SD}, \mathrm{n}=6)$. ${ }^{*}<0.001$ as revealed by the Mann-Whitney test. Vector, HepG2 cells transfected with pcDNA3.1 vector and cell clones Vector 1 and 2; HEPT3-AS, HepG2 cells transfected with HEPT3 antisense construct and cell clones HEPT3-AS1, -AS2, and -AS3.

(Fig. 5AIII). One was a 3211-bp human cDNA (GenBank AK130104), which contained the entire HEPT3 cDNA in its 3' region. The other was a 1743-bp full-length cDNA of human fetal brain (GenBank CR599670), which was 99\% homologous to the 3 ' portion of HEPT3 cDNA. On the
Northern blot, the $\sim 2.7 \mathrm{~kb}$ band with the strongest signal appeared to correspond to the full-length HEPT3 cDNA while the $\sim 3.3$ and $\sim 2 \mathrm{~kb}$ bands corresponded to the two isoforms identified in the database. Similar to HEPT3, both transcripts were encoded by intronless genomic DNA. These findings 
indicate that HEPT3 and its variants may be transcribed from the same genomic DNA.

HEPT3, a potential noncoding RNA. The NCBI ORFinder revealed a high density of stop codons in all the 6 theoretical reading frames (Fig. 5B). Although numerous start codons were scattered throughout the HEPT3 gene, the frequent stop codons resulted in a lack of an extensive open reading frame. Similar observations were obtained when the other two HEPT3 isoforms, the $3.2-\mathrm{kb}$ and $1.7-\mathrm{kb}$ transcripts, were analysed. These data suggest that HEPT3 may not possess coding capacity. However, searches with both the full-length and fragmented HEPT3 sequences failed to reveal any significant homology to the known microRNAs in the available databases.

Reduction of HepG2 cell growth rate by antisense HEPT3. As HEPT3 was markedly upregulated in HCC cell lines, we employed the antisense approach to interfere with the expression of HEPT3 in HepG2 cells and then to evaluate the involvement of HEPT3 in regulating cell growth. The antisense plasmid was constructed with a partial cDNA of HEPT3 in the antisense orientation (Fig. 6A). The Alu element was excluded to avoid binding to non-specific sequences. The endogenous HEPT3 mRNA levels were clearly decreased by antisense HEPT3 in three clones (Fig. 6D). Light microscopy (Fig. 6B) and MTT assay (Fig. 6C) revealed that the cell proliferation rate of these three cell clones was reduced $\sim 5$ fold on days 4 and $5(\mathrm{P}<0.001)$ when compared to the controls, two cell clones transfected with the empty vector and the parental HepG2 cells. In addition, disruption of HEPT3 reduced the colony formation of HepG2 cells. Not only did the antisense HEPT3 decrease the number of colonies by $>50 \%(\mathrm{P}<0.001)$ but also the size and cell density of the colonies (Fig. 6E). These data implicate that overexpressed HEPT3 exerts proliferative effect on the HCC cells.

\section{Discussion}

Using suppression subtractive hybridisation to compare the mRNA expression patterns of HCC and its adjacent nontumourous liver tissue, we identified a novel transcript, HEPT3, which is highly upregulated in $87 \%$ of 23 HCC tissues examined. However, no significant correlations could be observed between HEPT3 overexpression and the clinicopathological parameters in the $23 \mathrm{HCC}$ patients. This observation could be due to the high frequency (20/23) of HEPT3 overexpression in the patients and/or the relatively small sample size examined in this study. An elevated expression of HEPT3 was also detected in most (4/5) of the HCC cell lines tested, but no apparent overexpression of HEPT3 was observed in tissues and cell lines of other tumour types. The data indicate that HEPT3 overexpression is specific to HCC, suggesting that the gene may be associated with the tumorigenesis of hepatocytes. Liver-specific genes that are differentially expressed in HCC have been identified. For example, the human LFIRE-1/HFREP-1 is frequently downregulated or lost in HCC (10). Hopefully, the emerging of HEPT3 will shed new light on liver carcinogenesis.
The mechanism responsible for regulating HEPT3 expression is still elusive. The polyadenylated transcript of HEPT3 contains eleven copies of the mRNA destabilisation motif, AUUUA. Such motifs have been reported in lymphokines, cytokines and proto-oncogene mRNAs; and have been proposed to interact with the AU-binding factor that degrades mRNA (11). This finding offers a clue that the RNA levels of HEPT3 may be regulated by mechanisms involving RNA stability.

The gene HEPT3 encodes a transcript of $\sim 2.7 \mathrm{~kb}$ and displays several intriguing characteristics. The full-length cDNA of HEPT3 is collinear to its genomic DNA, indicating that HEPT3 represents an intronless gene. Naturally intronless genes account for $<6 \%$ of the genes in human (12). Since intronless genes do not require post-transcriptional splicing, they can be transcribed more efficiently leading to a potentially faster rate of expression, greater abundance and higher fidelity. Classic examples of intronless proto-oncogenes are the myc (13) and c-jun (14) genes. The lack of intron/exon boundaries in the genomic sequence implies that alternative splicing of HEPT3 is unlikely to occur. However, Northern blot analysis detected three differently sized transcripts, suggesting that HEPT3 has isoforms. Database analyses revealed two uncharacterised full-length cDNAs matching with HEPT3 both in sequence and in chromosomal location. These findings indicate that HEPT3 and its variants are transcribed from the same genomic DNA. However, the mechanism of generating these transcripts by the DNA machinery remains unclear.

Another striking feature of HEPT3 is the high density of stop codons in all the reading frames, resulting in a lack of extensive open reading frame. In addition, an Alu repetitive element was identified in the HEPT3 transcript. The presence of short interspersed repetitive sequences (SINEs) and other repeats is a frequent event in non-translatable transcripts (15), but rare in translated transcripts (16). Collectively, the atypical structural features of the cDNA suggest that HEPT3 may function as a noncoding RNA. Two examples of relatively well-studied mammalian genes that act at the RNA level are XIST (X (inactive)-specific transcript) and H19 $(17,18)$. XIST RNA coats one of the two $X$ chromosomes in females, resulting in transcriptional silencing of the $\mathrm{X}$ chromosome. $H 19$ has been implicated in imprinting of the insulin-like growth factor 2 gene important for tumour suppression (19). Moreover, many miRNAs (microRNAs), another group of noncoding RNAs, have been implicated in carcinogenesis including HCC (20-22). However, database searches failed to reveal significant homology or complementarities between HEPT3 sequences and the known miRNAs.

The gene HEPT3 is mapped to human chromosome 6q13-14. Chromosome 6 is best recognised for the major histocompatibility complex (MHC) that is critical to the human immune response. Additionally, it was documented to carry genes related to genetic diseases such as arthritis (23), diabetes (24), schizophrenia (25) and cancers. Gains in chromosome 6 have also been reported in HCC (26); however, it is unclear if HEPT3 is involved in such gains.

To determine if HEPT3 mRNA contributes to hepatocyte survival, we used an antisense inhibition approach to interfere with the biological activity of HEPT3. Suppression of the endogenous HEPT3 mRNA by antisense HEPT3 significantly 
inhibited both cell proliferation and colony formation of HepG2 cells, demonstrating that antisense HEPT3 exhibits antiproliferative effect on the HCC cells that express the endogenous HEPT3 gene. These results implicate that transcriptional activation of the HEPT3 gene may stimulate the oncogenic pathway that induces cell proliferation.

In conclusion, the gene HEPT3 is more commonly upregulated in HCC than in other tumour types. HEPT3 is an intronless gene and maps to chromosome 6q13-14. Its transcript displays the typical characteristics of noncoding RNA, including the lack of extensive ORF and the presence of an Alu sequence. Antisense studies revealed that the growth of HCC cells was significantly suppressed when HEPT3 expression level was reduced. The data indicate that HEPT3 may function through its noncoding RNA and play a role in the malignant transformation of hepatocytes. Further studies will be carried out to elucidate how HEPT3 transcription is activated in hepatocarcinogenesis and then promotes the progression of HCC.

\section{Acknowledgements}

This study was supported by the grant (No. R-185-000031-214) from the Yong Loo Lin School of Medicine at the National University of Singapore. We thank Ms. Asha Reka Das for her generous assistance.

\section{References}

1. Akriviadis EA, Llovet JM, Efremidis SC, Shouval D, Canelo R, Ringe B and Meyers WC: Hepatocellular carcinoma. Br J Surg 85: 1319-1331, 1998.

2. Kondoh N, Wakatsuki T, Ryo A, Hada A, Aihara T, Horiuchi S, et al: Identification and characterization of genes associated with human hepatocellular carcinogenesis. Cancer Res 59: 4990-4996, 1999.

3. Ng IO, Liang ZD, Cao L and Lee TK: DLC-1 is deleted in primary hepatocellular carcinoma and exerts inhibitory effects on the proliferation of hepatoma cell lines with deleted DLC-1. Cancer Res 60: 6581-6584, 2000.

4. Zhao X, Li J, He Y, Lan F, Guo J, Zhao R, et al: A novel growth suppressor gene on chromosome $17 \mathrm{p} 13.3$ with a high frequency of mutation in human hepatocellular carcinoma. Cancer Res 61: 7383-7387, 2001.

5. Zeng J, Wang H, Chen Z, Ullrich A and Wu M: Molecular cloning and characterization of a novel gene which is highly expressed in hepatocellular carcinoma. Oncogene 21: 4932-4943, 2002.

6. Tsou AP, Yang CW, Huang CY, Yu RC, Lee YC, Chang CW, et al: Identification of a novel cell cycle regulated gene, HURP, overexpressed in human hepatocellular carcinoma. Oncogene 22: 298-307, 2003.

7. Shao G, Zhou R, Zhang Q, Zhang Y, Liu, J, Rui J, et al: Molecular cloning and characterization of LAPTM4B, a novel gene upregulated in hepatocellular carcinoma. Oncogene 22: 5060-5069, 2003.
8. Moh MC, Lee LH and Shen S: Cloning and characterization of hepaCAM, a novel Ig-like cell adhesion molecule suppressed in human hepatocellular carcinoma. J Hepatol 42: 833-841, 2005.

9. Takeda K, Ichijo H, Fujii M, Mochida Y, Saitoh M, Nishitoh H, et al: Identification of a novel bone morphogenetic proteinresponsive gene that may function as a noncoding RNA. J Biol Chem 273: 17079-17085, 1998.

10. Yan J, Yu Y, Wang N, Chang Y, Ying H, Liu W, et al: LFIRE-1/ HFREP-1, a liver-specific gene, is frequently downregulated and has growth suppressor activity in hepatocellular carcinoma. Oncogene 23:1939-1949, 2004.

11. Shaw G and Kamen R: A conserved AU sequence from the 3' untranslated region of GM-CSF mRNA mediates selective mRNA degradation. Cell 46: 659-667, 1986.

12. Lewin B: Genes VII. Oxford University Press, New York, 2000.

13. Sugiyama A, Noguchi K, Kitanaka C, Katou N, Tashiro F, Ono T, et al: Molecular cloning and chromosomal mapping of mouse intronless myc gene acting as a potent apoptosis inducer. Gene 226: 273-283, 1999

14. Hattori K, Angel P, Le Beau MM and Karin M: Structure and chromosomal localization of the functional intronless human JUN protooncogene. Proc Natl Acad Sci USA 85: 9148-9152, 1988.

15. Michel U: Non-coding ribonucleic acids - a class of their own? Int Rev Cytol 218: 143-219, 2002.

16. Yulug IG, Yulug A and Fisher EM: The frequency and position of Alu repeats in cDNAs, as determined by database searching. Genomics 27: 544-548, 1995.

17. Szymanski M, Barciszewska MZ, Zywicki M and Barciszewski J: Noncoding RNA transcripts. J Appl Genet 44: 1-19, 2003.

18. Askew DS and Xu F: New insights into the function of noncoding RNA and its potential role in disease pathogenesis. Histol Histopathol 14: 235-241, 1999.

19. Okamoto K, Morison IM, Taniguchi T and Reeve AE: Epigenetic changes at the insulin-like growth factor II/H19 locus in developing kidney is an early event in Wilms tumorigenesis. Proc Natl Acad Sci USA 94: 5367-5371, 1997.

20. Hede K: Studies define role of microRNA in cancer. J Natl Cancer Inst 97: 1114-1115, 2005.

21. Gregory RI and Shiekhattar R: MicroRNA biogenesis and cancer. Cancer Res 65: 3509-3512, 2005.

22. Murakami Y, Yasuda T, Saigo K, Urashima T, Toyoda H, Okanoue T and Shimotohno K: Comprehensive analysis of microRNA expression patterns in hepatocellular carcinoma and non-tumorous tissues. Oncogene 25: 2537-2545, 2006.

23. Jawaheer D, Seldin MF, Amos CI, Chen WV, Shigeta R, Etzel C, et al: North American Rheumatoid Arthritis Consortium. Screening the genome for rheumatoid arthritis susceptibility genes: a replication study and combined analysis of 512 multicase families. Arthritis Rheum 48: 906-916, 2003.

24. Metz C, Cave H, Bertrand AM, Deffert C, Gueguen-Giroux B, Czernichow P, et al: NDM French Study Group. Neonatal diabetes mellitus. Neonatal diabetes mellitus: chromosomal analysis in transient and permanent cases. J Pediatr 141: 483-489, 2002.

25. Lindholm E, Ekholm B, Balciuniene J, Johansson G, Castensson A, Koisti M, et al: Linkage analysis of a large Swedish kindred provides further support for a susceptibility locus for schizophrenia on chromosome 6p23. Am J Med Genet 88: 369-377, 1999.

26. Zimmermann U, Feneux D, Mathey G, Gayral F, Franco D and Bedossa P: Chromosomal aberrations in hepatocellular carcinomas: relationship with pathological features. Hepatology 26: 1492-1498, 1997. 